\title{
Evodiamine activates autophagy as a cytoprotective response in murine Lewis lung carcinoma cells
}

\author{
YONG-JIU TU ${ }^{1,2}$, XIA FAN $^{1}$, XUE YANG ${ }^{1}$, CHUN ZHANG $^{1}$ and HUA-PING LIANG ${ }^{1}$ \\ ${ }^{1}$ State Key Laboratory of Trauma, Burns and Combined Injury, Research Institute of Surgery, Daping Hospital, \\ The Third Military Medical University, Chongqing 400042; ${ }^{2}$ Department of General Surgery, \\ 174th Hospital of PLA, Xiamen, Fujian 361003, P.R. China
}

Received July 10, 2012; Accepted August 24, 2012

DOI: $10.3892 /$ or.2012.2125

\begin{abstract}
Autophagy is a self-defense mechanism that provides nutrition and energy for cell survival by recycling the cytoplasm and organelles. Hence, chemotherapy is rendered less effective against cancer cells. Evodiamine is a previously described biological agent that possesses a cytotoxic activity in multiple cancer cells. However, little is known about evodiamine-induced autophagy in Lewis lung carcinoma (LLC) cells. In this study, LLC cells and a xenograft model were used. By use of a panel of techniques such as MTT assay, flow cytometry, western blotting, immunocytochemistry and TUNEL assay, the effects on the induction of apoptosis and autophagy were evaluated. We demonstrated that evodiamine inhibited LLC cell growth and induced apoptosis through caspase-independent manner in vitro and caspase-dependent pathway in vivo. In addition, we showed for the first time that evodiamine promoted autophagosome formation by enhancing the conversion of microtubule-associated protein 1 light chain 3 (LC3)-I to LC3-II and upregulating the expression of autophagy-specific genes (Atgs). Moreover, 3-methyladenine (3-MA), an autophagy inhibitor, attenuated evodiamineinduced autophagy through decreasing the conversion of LC3-I to LC3-II. The inhibition of autophagy was found to increase cell death and enhance evodiamine-induced apoptosis in vitro in a caspase-independent manner and in vivo in a caspase-dependent manner. In conclusion, evodiamine promoted autophagy in LLC cells and autophagy inhibition
\end{abstract}

Correspondence to: Professor Hua-Ping Liang or Professor Chun Zhang, State Key Laboratory of Trauma, Burns and Combined Injury, Research Institute of Surgery, Daping Hospital, The Third Military Medical University, 10 Changjiang Road, Daping, Chongqing 400042, P.R. China E-mail: huaping_liang@yahoo.com.cn

E-mail: zhangchun.ma@yahoo.com.cn

Abbreviations: LLC, Lewis lung carcinoma; Atg, autophagy-specific gene; MDC, monodansylcadaverine; LC3, microtubule-associated protein 1 light chain 3; 3-MA, 3-methyladenine; AIF, apoptosisinducing factor; endo G, endonuclease G; PI3K, phosphatidylinositol 3 kinase

Key words: evodiamine, autophagy, apoptosis, Lewis lung carcinoma enhanced evodiamine-induced apoptosis in vitro and in vivo. These results demonstrate that evodiamine-induced autophagy plays a cytoprotective role in LLC cells and evodiamine combined with autophagy inhibitor therapy could increase the chemosensitivity of LLC cells.

\section{Introduction}

Lung cancer is the most commonly diagnosed cancer worldwide and is the leading cause of cancer-related mortality among men and women (1). Chemotherapy remains one of the primary modalities for treating lung cancer. However, the clinical use of cytotoxic drugs is limited due to intrinsic or acquired resistance and toxicity (2). Despite the improvements in cancer therapy over the last 30 years, the overall 5-year survival rate is generally less than $15 \%$ (3). To improve the survival rate, intensive efforts have been made to find new anticancer agents.

Evodiamine is one of the major bioactive components derived from $\mathrm{Wu}-\mathrm{Chu}-\mathrm{Yu}$, a long-standing Chinese herb. Evodiamine has been reported to possess various biological activities, including anti-inflammatory effects $(4,5)$, and to have inhibitory effects on adipogenesis (6) and gastrointestinal disorders (7). Evodiamine is effective for inducing cell cycle arrest and apoptosis in human colon LoVo cells (8), cervical HeLa cells (9), melanoma A375-S2 cells (10), thyroid cancer cells (11), hepatocellular SMMC-7721 cells (12) and prostate DU145 and PC3 cell lines (13). Moreover, evodiamine inhibited tumor cell migration in murine Lewis lung carcinoma (LLC) cells (14). However, the effect of evodiamine on LLC cell growth and death remains unclear.

The study of autophagic activity and its contribution to the development of cancer has gained interest in recent years. Autophagy is an evolutionary conserved eukaryotic process in which organelles and bulk proteins are turned over by lysosomal activity. The physiologic function of autophagy is to maintain homeostasis by eliminating unnecessary proteins and injured or aged organelles. These processes are regulated by a group of autophagy-specific genes (Atgs) which function to sense environmental stress, assemble double- or multi-membrane-bound autophagic vacuoles to sequester the cytoplasmic materials (termed autophagosome), and facilitate the transport of autophagosomes to lysosomes for degradation (15). Autophagy has different effects on cell survival depending 
on the stage of cell development (16). It has been reported that autophagy displays a suppression effect at the initial stage, in which tumor growth is rapid, or may be required to provide essential nutrients to the cells in advanced stages of cancer (17). Thus, autophagy is not only involved in cell death, it can also induce recycling of proteins and organelles to sustain cell survival. However, this self-defense mechanism can render cancer cells insensitive to anticancer agents. This can be overturned by inhibiting autophagy, whereby autophagy inhibition could potentiate cisplatin-induced apoptosis in esophageal squamous carcinoma cells (18), upregulate anthocyanininduced apoptosis in hepatocellular carcinoma cells (19) and augment 5-fluorouracil chemotherapy in human colon cells in vitro and in vivo $(20,21)$. Furthermore, evodiamine stimulated autophagy in human cervical HeLa cells as a survival function and an autophagy inhibitor enhanced the sensitivity of HeLa cells to evodiamine (22). It is unclear whether evodiamine can induce autophagy in LLC cells, or whether evodiamine-induced autophagy in LLC cells is cytoprotective.

In this study, we examined the anticancer activity of evodiamine in LLC cells. We demonstrated that evodiamine inhibited proliferation of LLC cells and increased apoptosis in vitro and in vivo. Evodiamine induced autophagy by converting microtubule-associated protein 1 light chain 3 (LC3)-I to LC3-II and expressions of Atgs. Inhibiting autophagy enhanced evodiamine-induced apoptosis in vitro in a caspase-independent manner and in vivo in a caspasedependent manner. Overall, evodiamine-induced autophagy may play a cytoprotective role in LLC cells, and evodiamine combined with an autophagy inhibitor therapy could attenuate the chemoresistance of LLC cells.

\section{Materials and methods}

Animals. All animals used in our study were athymic mice, not human or non-human primates. Animals were used to establish a murine Lewis lung carcinoma xenograft model through subcutaneously injecting cells and agents for the treatment were administered intratumorally every 3 days for a total of 5 times. All efforts were made to minimize suffering and to provide appropriate conditions, including a sterile environment and room temperature at $24^{\circ} \mathrm{C}$, sterile distilled water, eggs and apples.

Cell line and cell culture. The murine Lewis lung carcinoma cell line (LLC) was obtained from the State Key Laboratory of Trauma, Burns and Combined Injury, Research Institute of Surgery (Chongqing, China). The cells were cultured in Dulbecco's modified Eagle's medium (DMEM) containing $10 \%$ fetal bovine serum (FBS), $100 \mathrm{U} / \mathrm{ml}$ penicillin, and $100 \mu \mathrm{g} / \mathrm{ml}$ streptomycin at $37^{\circ} \mathrm{C}$ in the presence of $95 \%$ air, $5 \%$ $\mathrm{CO}_{2}$ with medium change every 2 days. Cells in the exponential growth phase were harvested for use.

Chemicals and antibodies. Evodiamine was purchased from Xi'an Guanyu Bio-Tech Co., Ltd. (Xi'an, China) with a purity of $98 \%$ determined by HPLC. Evodiamine was dissolved in dimethylsulphoxide (DMSO) and diluted with DMEM prior to use. The DMSO concentration in the cell culture medium was $<0.1 \%$.
DMEM and FBS were obtained from Gibco BRL (Gaithersburg, MD, USA). DMSO, MTT (3-(4,5-dimethylthiazole-2-yl)-2, 5-diphenyltetrazolium bromide), 3-methyladenine (3-MA), monodansylcadaverine (MDC) and 4',6-diamidino-2-phenylindole (DAPI) were purchased from Sigma-Aldrich (St. Louis, MO, USA). Annexin V-FITC/PI apoptosis detection kit was from KeyGen Biotech (Nanjing, China). Chemiluminescence kit and BCA protein assay kit were from KangChen Bio-tech (Shanghai, China). TUNEL apoptosis detection kit was obtained from Roche Applied Science (Indianapolis, IN, USA). Athymic mice were from the Chinese Academy of Sciences (Shanghai, China). The following primary antibodies were used in this study: LC3, Atg3, Atg4b, Atg5 and Atg7 (Sigma-Aldrich), caspase-3 (Fisher Scientific, Hudson, NH, USA).

MTT assay and cell morphological assay. A modified MTT assay was employed to quantify the cell proliferation. LLC cells were seeded at a density of $1 \times 10^{4} /$ well in 96 -well plates. Following incubation with agents of different concentrations for the indicated times, $20 \mu \mathrm{l}$ of $5 \mathrm{mg} / \mathrm{ml}$ MTT solution were added to each well followed by incubation for $6 \mathrm{~h}$. Then, $50 \mu \mathrm{l}$ of $20 \%$ SDS solution were added in each well followed by incubation overnight. The optical density was determined with a microplate reader (BioTek ELx800, USA) at $570 \mathrm{~nm}$ and 50\% inhibition of cell growth $\left(\mathrm{IC}_{50}\right)$ was evaluated. The percentage of cell viability was calculated as follows: Cell viability $(\%)=$ $\mathrm{A}_{570}$ (evodiamine) $/ \mathrm{A}_{570}$ (control) x $100 \%$.

LLC cells at a density of $1 \times 10^{5} /$ well were seeded in 6-well plates and maintained overnight. The medium was discarded and replaced with fresh medium or medium containing evodiamine of different concentrations for $48 \mathrm{~h}$. Cell morphology was observed and representative images were captured under a light microscope.

Flow cytometry. Apoptosis was measured by detecting phosphatidylserine that was exposed on cell membranes using an apoptosis detection kit. LLC cells treated with indicated agents at indicated times were harvested and washed with PBS, then resuspended in staining solution containing PI $(50 \mu \mathrm{g} / \mathrm{ml})$ and Annexin V-FITC $(25 \mu \mathrm{g} / \mathrm{ml})$ followed by incubation for $15 \mathrm{~min}$ at room temperature in the dark. Cells were resuspended in the binding buffer and apoptotic cells were analyzed on a flow cytometry system (FACScan; Becton-Dickinson, Heidelberg, Germany). Data were analyzed using CellQuest software (Becton-Dickinson).

Analysis of autophagy. A fluorescent compound, MDC, has been proposed as a special tracer for autophagic vacuoles. Treated and untreated cells were harvested and washed with PBS, then resuspended in $0.05 \mathrm{mM} \mathrm{MDC}$ and incubated at $37^{\circ} \mathrm{C}$ for $30 \mathrm{~min}$. Following incubation, cells were washed with PBS and cell number was counted under a light microscope. Then, cells were lysed with lysis buffer $(1 \mathrm{mM}$ Tris- $\mathrm{HCl}$, $1 \%$ Triton X-100, pH 7.5) at room temperature in the dark. Cell lysate was added into 96-well plates at a density of $100 \mu \mathrm{l} /$ well and MDC (excitation wavelength $325 \mathrm{~nm}$, emission filter $525 \mathrm{~nm}$ ) fluorescence in cell lysate was determined with Optima FLUOstar plate reader (BMG Labtech, Durham, NC, USA). The amount of autophagic vacuoles in single cells was 
evaluated by obtaining the ratio of MDC fluorescence to the amount of cells. Also, the autophagic vacuoles of cells growing on cover-slips were labeled with MDC at $37^{\circ} \mathrm{C}$ for $30 \mathrm{~min}$ and then cell nuclei were stained with DAPI. Following incubation, cells were washed with PBS and representative images were captured under a laser confocal scanning microscope (Nikon Eclipse TE 300, Germany).

Western blot assay. Treated or untreated cells were harvested and washed twice in ice-cold PBS, then lysed with lysis buffer. Cell lysate was centrifuged at $13,000 \mathrm{~g}$ for $5 \mathrm{~min}$ at $4^{\circ} \mathrm{C}$. The protein concentration was determined by a BCA protein assay kit. Protein was loaded onto SDS-PAGE gel and electro-transferred to polyvinylidene fluoride membranes. After blocking with $5 \%$ non-fat milk, the membranes were incubated with specific primary antibodies followed by horseradish peroxidase conjugated secondary antibodies. Finally, the membranes were visualized with an enhanced chemiluminescence kit. Signal intensity was quantified by densitometric analysis (Tanon, Shanghai, China).

In vivo antitumor effect. A total of 32 female athymic mice aged 4-6 weeks were given ad libitum access to sterilized food and water. Mice were subcutaneously administered $2 \times 10^{6}$ of LLC cells in $0.1 \mathrm{ml}$ of PBS. Two weeks later, when the tumor size reached $\sim 100 \mathrm{~mm}^{3}$, mice were randomly divided into the DMSO, 3-MA, evodiamine and evodiamine + 3-MA groups ( $\mathrm{n}=8$ per group). Evodiamine $(1 \mathrm{mg} / \mathrm{kg})$ and/or $25 \mathrm{mg} / \mathrm{kg}$ 3-MA dissolved in $100 \mu \mathrm{l}$ DMSO was injected intratumorally every 3 days for a total of 5 times (Days 15, 18, 21, 24 and 27) and $100 \mu \mathrm{l}$ DMSO were injected as the control. The tumor volume and body weight were measured during the experimental period, and tumor volume was determined by the following formula: $0.5 \mathrm{xLxW}^{2}$ (L, length; $\mathrm{W}$, width). The animals were sacrificed after five times of measurement and tumors were removed. A section of the tumor was fixed in $4 \%$ paraformaldehyde, embedded in paraffin, cut into $5-\mu \mathrm{m}$ sections and mounted on slides for further analysis; another section was stored at $-70^{\circ} \mathrm{C}$ for western blot analysis.

Apoptosis in tumors. Apoptosis was measured by a TUNEL staining kit according to the manufacturer's instructions. The sections were deparaffinized in xylene, and dehydrated in gradient ethanol. The sections were permeabilized with $20 \mu \mathrm{g} / \mathrm{ml}$ protease for $30 \mathrm{~min}$ at $37^{\circ} \mathrm{C}$ followed by $50 \mu 1$ of TUNEL reaction mixture for $60 \mathrm{~min}$ at $37^{\circ} \mathrm{C}$, and further analyzed using a fluorescence microscope.

Statistical analysis. Data are presented as means \pm standard deviation (SD). Statistical analysis was performed with one-way analysis of variance (ANOVA) or Student's t-test for independent variables. $\mathrm{P}<0.5$ was considered to indicate statistically significant differences.

\section{Results}

Evodiamine inhibits cell proliferation. The effect of evodiamine on the growth of LLC cells was measured by MTT assay. LLC cells were seeded and treated with evodiamine $(7.5-60 \mu \mathrm{M})$ for 1-3 days. After $24 \mathrm{~h}$ of treatment, evodiamine modestly
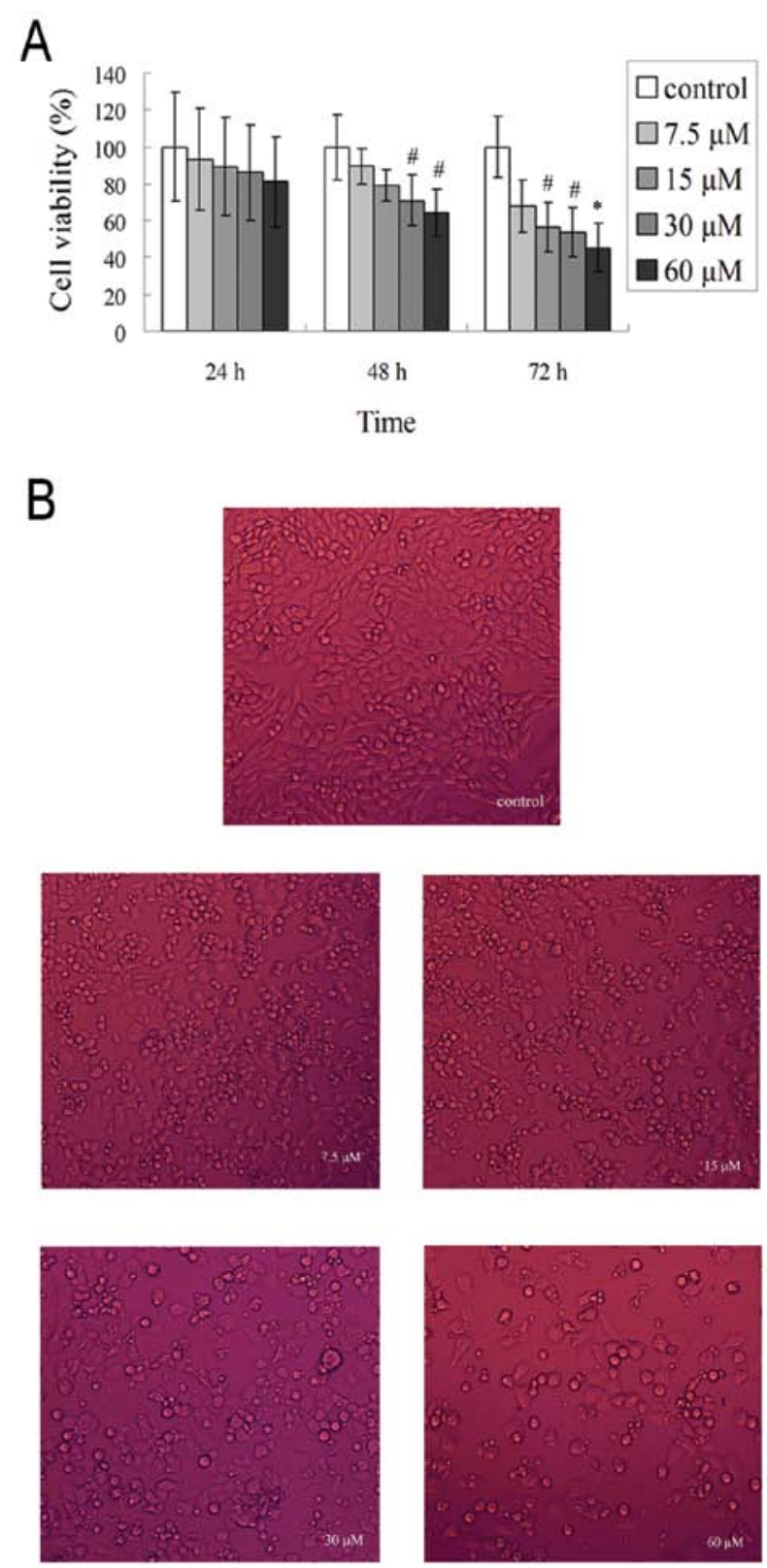

Figure 1. Evodiamine inhibits LLC cell proliferation. (A) LLC cells were grown in 96 -well plates $\left(1 \times 10^{4}\right.$ cells/well) and treated with different doses of evodiamine treatment for the indicated time periods. The survival rate was measured by MTT assay and presented as the percentage by control, which was defined as $100 \%$. Data are expressed as means $\pm \mathrm{SD} ;(\mathrm{n}=5) ;{ }^{*} \mathrm{P}<0.01$ and ${ }^{\#} \mathrm{P}<0.05$ vs. the control group. (B) LLC cells were treated with different doses of evodiamine for $48 \mathrm{~h}$. Morphological changes were captured by light microscopy (x200).

decreased the proliferation of LLC cells. However, evodiamine $\left(60 \mu \mathrm{M}, \mathrm{IC}_{50}=113 \mu \mathrm{M}\right)$ significantly inhibited the growth of LLC cells at $48 \mathrm{~h}$. Furthermore, the viability of evodiaminetreated cells after 72 -h treatment was significantly decreased $\left(60 \mu \mathrm{M}, \mathrm{IC}_{50}=38 \mu \mathrm{M}\right)$ (Fig. 1A). These results showed that evodiamine inhibited the proliferation of LLC cells in a timeand dose-dependent manner. Evodiamine-treated cells also displayed noticeable morphological changes characterized by decreased cell density and smaller cell conjunctions. Finally, cells became round, floating and cell debris was found in the $60 \mu \mathrm{M}$ evodiamine treatment group (Fig. 1B). 


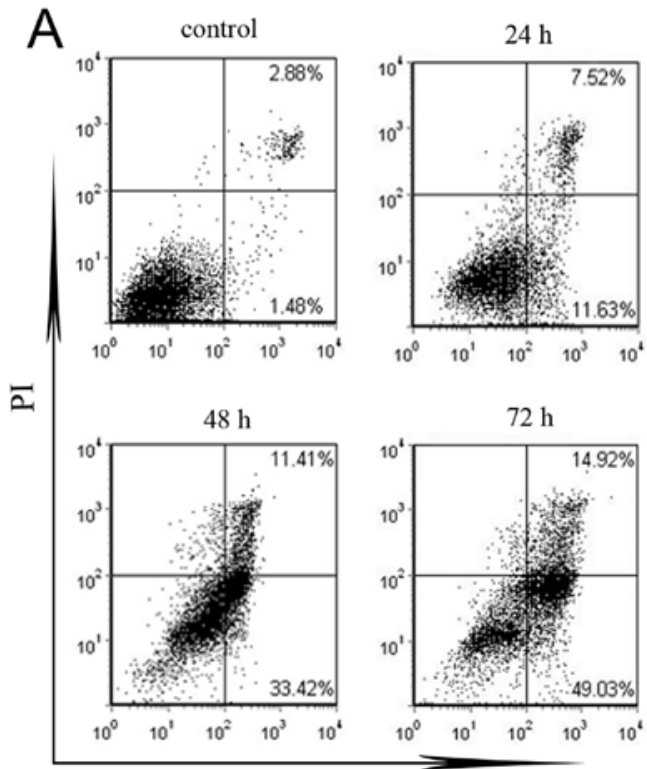

Annexin V-FITC

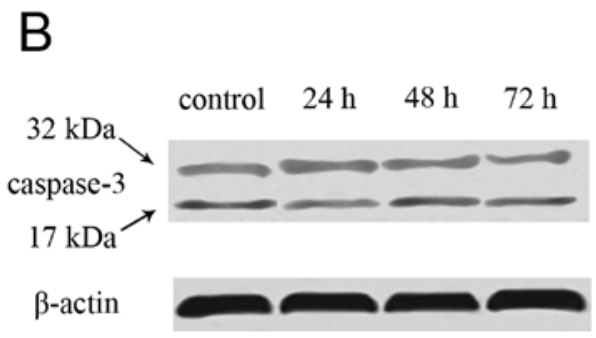

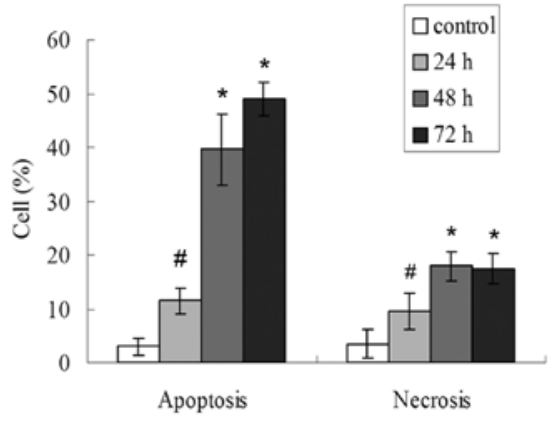

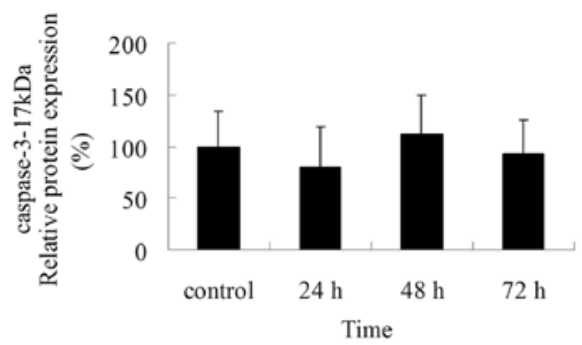

Figure 2. Evodiamine induces caspase-independent apoptosis. (A) LLC cells were treated with $60 \mu \mathrm{M}$ evodiamine for 24,48 and $72 \mathrm{~h}$. Evodiamine-induced early apoptosis was observed by flow cytometry. Data are presented as means $\pm \mathrm{SD},(\mathrm{n}=3) .{ }^{*} \mathrm{P}<0.01$ and ${ }^{\text {\# }} \mathrm{P}<0.05$ vs. the control group. (B) LLC cells were treated with $60 \mu \mathrm{M}$ evodiamine for 24,48 and $72 \mathrm{~h}$. Total protein was isolated and subjected to western blot analysis for caspase-3 protein expression. $\beta$-actin was used as a loading control. Data are expressed as percentage and presented as means $\pm \mathrm{SD}$. $\mathrm{P}<0.01$ and ${ }^{*} \mathrm{P}<0.05$ vs. the control group.
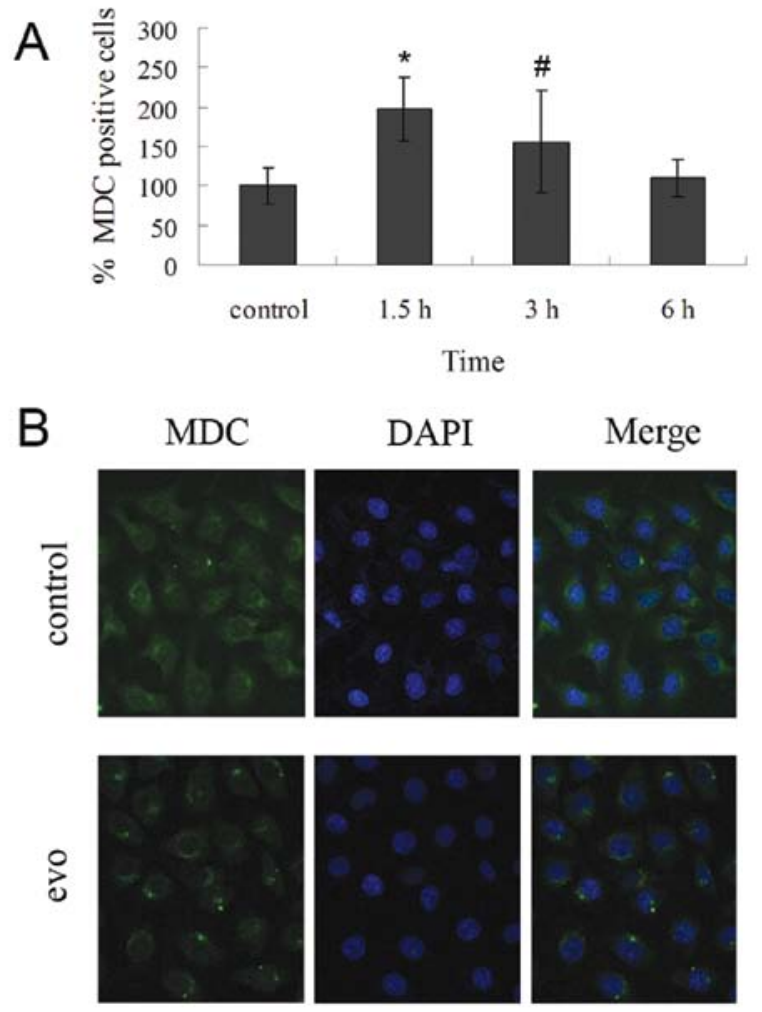

Evodiamine induces caspase-independent apoptosis. We further assessed whether evodiamine could induce apoptosis of LLC cells by flow cytometry. By means of Annexin V labeling to phosphatidylserine, evodiamine-treated LLC cells underwent early apoptosis (Annexin V-FITC-positive and PI-negative), which was increased to $11.5 \%$ in 24-h treatment and persisted to $72 \mathrm{~h}$ (Fig. 2A). Necrosis (Annexin V-FITC positive and PI-positive) was modestly observed in a time-dependent manner (Fig. 2A). However, the apoptotic mechanism was caspaseindependent as evodiamine-treated LLC cells did not display cleaved caspase-3 (17 kDa) (Fig. 2B).

Figure 3. Evodiamine promotes autophagy in LLC cells. (A) LLC cells were labeled with $0.05 \mathrm{mM} \mathrm{MDC}$ for $30 \mathrm{~min}$ at $37^{\circ} \mathrm{C}$ after treatment with $60 \mu \mathrm{M}$ evodiamine for $1.5,3$ and $6 \mathrm{~h}$. The cell number was then counted under a light microscope and cells were lysed with lysis buffer. Cell lysate was added into 96-well plates at a density of $100 \mu \mathrm{l} /$ well and MDC (excitation wavelength $325 \mathrm{~nm}$, emission filter $525 \mathrm{~nm}$ ) fluorescence in cell lysate was determined with the Optima FLUOstar plate reader. Data are expressed as means \pm SD, $(\mathrm{n}=3)$. ${ }^{*} \mathrm{P}<0.01$ and ${ }^{\#} \mathrm{P}<0.05$ vs. the control group. (B) LLC cells were treated with $60 \mu \mathrm{M}$ evodiamine for $1.5 \mathrm{~h}$ and incubated with $0.05 \mathrm{mM}$ MDC for $30 \mathrm{~min}$ at $37^{\circ} \mathrm{C}$, and the nucleus was then labeled with DAPI. MDC fluorescence was observed by laser confocal scanning microscope. Representative images are displayed $(\mathrm{x} 400)$. 


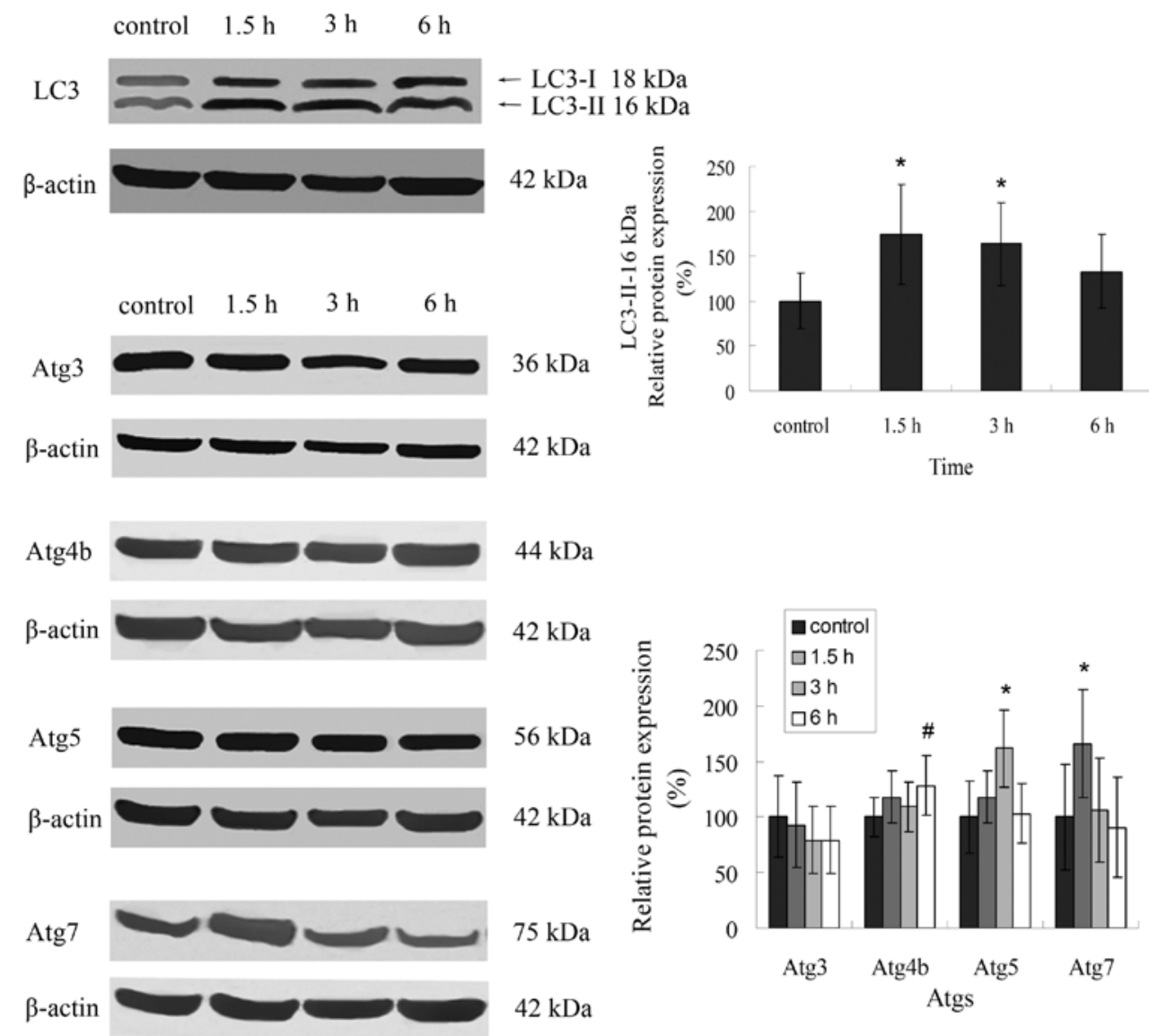

Figure 4. LC3 is involved in evodiamine-induced autophagy. LLC cells were treated with $60 \mu \mathrm{M}$ evodiamine for 1.5, 3 and $6 \mathrm{~h}$. Total protein was isolated and subjected to western blot analysis for LC3, Atg3, Atg4b, Atg5 and Atg7 protein expressions. $\beta$-actin was used as an equal loading control. Data are expressed as means $\pm \mathrm{SD},(\mathrm{n}=3)$. $* \mathrm{P}<0.01$ and ${ }^{\#} \mathrm{P}<0.05$ vs. the control group.

Evodiamine promotes autophagy in LLC cells. A fluorescent compound, MDC, was used to label autophagic vacuoles and changes in autophagic activity. LLC cells exhibited an increase in MDC fluorescence within 1.5-6 $\mathrm{h}$ after evodiamine treatment, while the peak of autophagy activity was observed in 1.5-h treatment (Fig. 3A). In addition, evodiamine-treated LLC cells displayed a greater number of distinct spots within the cytoplasm or perinuclear regions compared to control (Fig. 3B).

LC3 is involved in evodiamine-induced autophagy. LC3 is widely used as a molecular marker protein for autophagy. Evodiamine-treated LLC cells markedly upregulated LC3 expression with concomitant conversion of LC3-I to LC3-II, and the peak of expression was at 1.5 -h treatment, which was consistent with our previous results (Fig. 4). LC3 conversion as well as autophagosome formation are regulated by Atgs including Atg3, Atg4b, Atg5 and Atg7. Accordingly, evodiamine treatment increased the expression of Atg $4 \mathrm{~b}$, Atg5 and Atg7 in LLC cells. Evodiamine had no effects on Atg3 expression (Fig. 4).

Inhibition of autophagy enhances evodiamine-induced cell death. Evodiamine-induced autophagy has previously been detected in LLC cells. To clarify the relevance of autophagy in the cytotoxicity of evodiamine, we used an autophagy inhibitor, 3-MA, that inhibits the activity of class III phosphatidylinositol 3 kinase (PI3K). There was a 1.98-fold increase in the incorporation of MDC in evodiamine-treated LLC cells compared to control-treated groups. The addition of 3-MA together with evodiamine decreased MDC incorporation by 1.2 -fold (Fig. 5A). Furthermore, combination treatment with 3-MA and the evodiamine decreased the number of spots compared to evodiamine alone group (Fig. 5B). Then, molecular changes were detected during the following experiment. The combination treatment also decreased the expression of LC3 and the conversion of LC3-I to LC3-II, which were sharply increased by $60 \mu \mathrm{M}$ evodiamine for $1.5 \mathrm{~h}$. Moreover, upregulated expressions of Atg5 and Atg7 induced by evodiamine were decreased by 3-MA, but had no effect on Atg4 (Fig. 6A).

Autophagy has previously been reported to increase cell survival. Furthermore, the inhibition of autophagy contributes to enhanced cytotoxicity of chemotherapy in cancer cells. We examined the effects of evodiamine-induced autophagy inhibition on the cell death of LLC cells. Evodiamine induced cell death of LLC cells in a concentration- or time-dependent manner as assessed by MTT assay (Fig. 1A). Combination treatment with 3-MA and evodiamine greatly enhanced cell death. Cell viabilities were decreased to $<35 \%$ at 48 -h treatment and $10 \%$ at 72 -h treatment. A low concentration of 3-MA did not induce cell death at 48 or $72 \mathrm{~h}$ post-treatment (Fig. 6B). These observations suggest that 3-MA inhibited evodiamine-induced autophagy, but enhanced evodiamine-induced cell death.

3-MA augments evodiamine-induced apoptosis in LLC cells. We next evaluated the effects of 3-MA and evodiamine combi- 

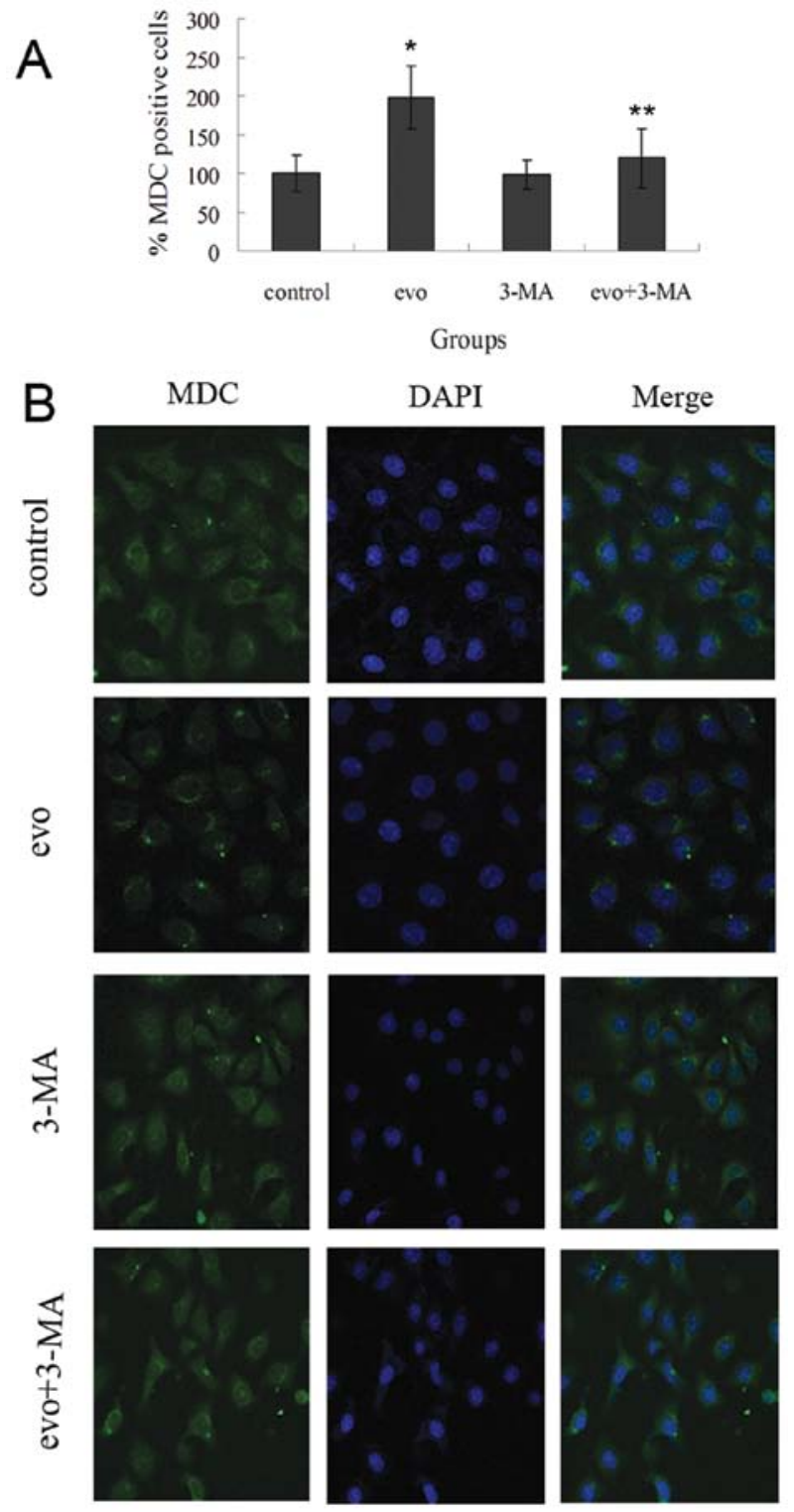

Figure 5. 3-MA inhibited evodiamine-induced autophagy. (A) LLC cells were labeled with $0.05 \mathrm{mM} \mathrm{MDC}$ for $30 \mathrm{~min}$ at $37^{\circ} \mathrm{C}$ after treatment with the indicated agents for $1.5 \mathrm{~h}$. Then, the cell number was counted under a light microscope and cells were lysed with lysis buffer. Cell lysate was added into 96-well plates at a density of $100 \mu 1 /$ well and MDC (excitation wavelength $325 \mathrm{~nm}$, emission filter $525 \mathrm{~nm}$ ) fluorescence in cell lysate was determined with the Optima FLUOstar plate reader. Data are expressed as means $\pm \mathrm{SD},(\mathrm{n}=3) .{ }^{*} \mathrm{P}<0.01$ vs. the control group, ${ }^{* * *} \mathrm{P}<0.01$ vs. the evodiamine treatment group. (B) LLC cells were treated with the indicated agents for $1.5 \mathrm{~h}$ and incubated with $0.05 \mathrm{mM} \mathrm{MDC}$ for $30 \mathrm{~min}$ at $37^{\circ} \mathrm{C}$, and the nucleus was labeled with DAPI. MDC fluorescence was observed by laser confocal scanning microscope. Representative images are displayed (x400).

national treatment on cell apoptosis. Evodiamine alone was able to induce apoptosis, while 3-MA treatment was able to enhance evodiamine-induced cell apoptosis. Necrosis was also moderately increased in the combinatorial treatment (Fig. 7A). Furthermore, consistent with the evodiamine alone group, the combinatorial treatment had no effect on the active form of caspase-3 (17 kDa) (Fig. 7B).

In vivo effects of evodiamine and $3-M A$. To determine the effects of evodiamine and 3-MA in vivo, mice were subcutaneously administered LLC cells, and were then treated with DMSO,
3-MA, evodiamine and evodiamine + 3-MA, respectively. In the final measurement, the tumor volume in the evodiamine alone group was smaller than in the control group. Furthermore, decreased tumor volume was greatest with the combinatorial treatment using evodiamine and 3-MA (Fig. 8A and B). The tumor growth curve revealed that treatment with evodiamine alone suppressed tumor growth with further inhibitory effects using the combinatorial treatment (Fig. 8C). Notably, the treatment of 3-MA alone also inhibited tumor growth that was comparable to the evodiamine treatment. No changes in body weight or death were noted during the time of study (Fig. 8D).

Evodiamine and 3-MA combinatorial treatment enhances caspase-dependent apoptosis in vivo. Our results indicated that 3-MA inhibited evodiamine-induced autophagy and enhanced caspase-independent apoptosis in vitro. Moreover, we found that evodiamine combined with 3-MA significantly inhibited tumor growth in LLC xenografts. Histological staining showed that evodiamine as well as the combinatorial treatment led to shrinkage of the cell nucleus, pyknosis and karyorrhexis. No effect on cell morphology was observed with the treatment of 3-MA alone. Increased number of TUNEL-positive cells was observed in all treated groups compared to the control. Furthermore, this increasing effect of evodiamine and 3-MA combination treatment was the greatest (Fig. 9A). To determine the molecular mechanism of apoptosis, the expression of cleaved caspase-3 $(17 \mathrm{kDa})$ in tumor tissue was examined. Contrary to the in vitro study, both the evodiamine and 3-MA alone groups weakly increased the protein level of cleaved caspase-3 $(17 \mathrm{kDa})$, while evodiamine and 3-MA combination significantly induced increasing cleaved caspase- 3 protein levels (Fig. 9B). Moreover, evodiamine slightly enhanced the conversion of LC3-I to LC3-II which was slightly attenuated in the combinatorial treatment (Fig. 9C).

\section{Discussion}

Lung cancer is the leading cause of cancer-related mortality and effective treatment is needed. Evodiamine, a bioactive component of $\mathrm{Wu}-\mathrm{Chu}-\mathrm{Yu}$, is effective for treating gastrointestinal disorders (7), and possesses anti-inflammatory activity $(4,5)$ and inhibitory effects on adipogenesis $(6)$. Evodiamine also possesses anticancer properties by inhibiting the growth of various cancer cells (8-13). In this study, we investigated its anticancer effects against murine Lewis lung carcinoma cells. We showed by flow cytometry and TUNEL staining that evodiamine induces apoptosis of LLC cells in vitro and in vivo.

Targeting the apoptotic pathway is of great therapeutic interest for novel targeted therapies to induce cancer cell death or sensitize them to established cytotoxic agents (23). The caspase-dependent pathway is a classical apoptotic pathway. This includes the extrinsic and intrinsic pathways for the activation of caspase- 8 and -9 , respectively, followed by the caspase-3 (24). Our study illustrates that evodiamine-induced apoptosis in LLC cells was caspase-independent in vitro. This indicates that other apoptotic mechanisms are executed, such as those that are mediated by apoptosis-inducing factor (AIF) and endonuclease $G$ (endo $G$ ) (25). Whether this mechanism is involved in evodiamine-induced apoptosis of LLC cells remains to be determined. 

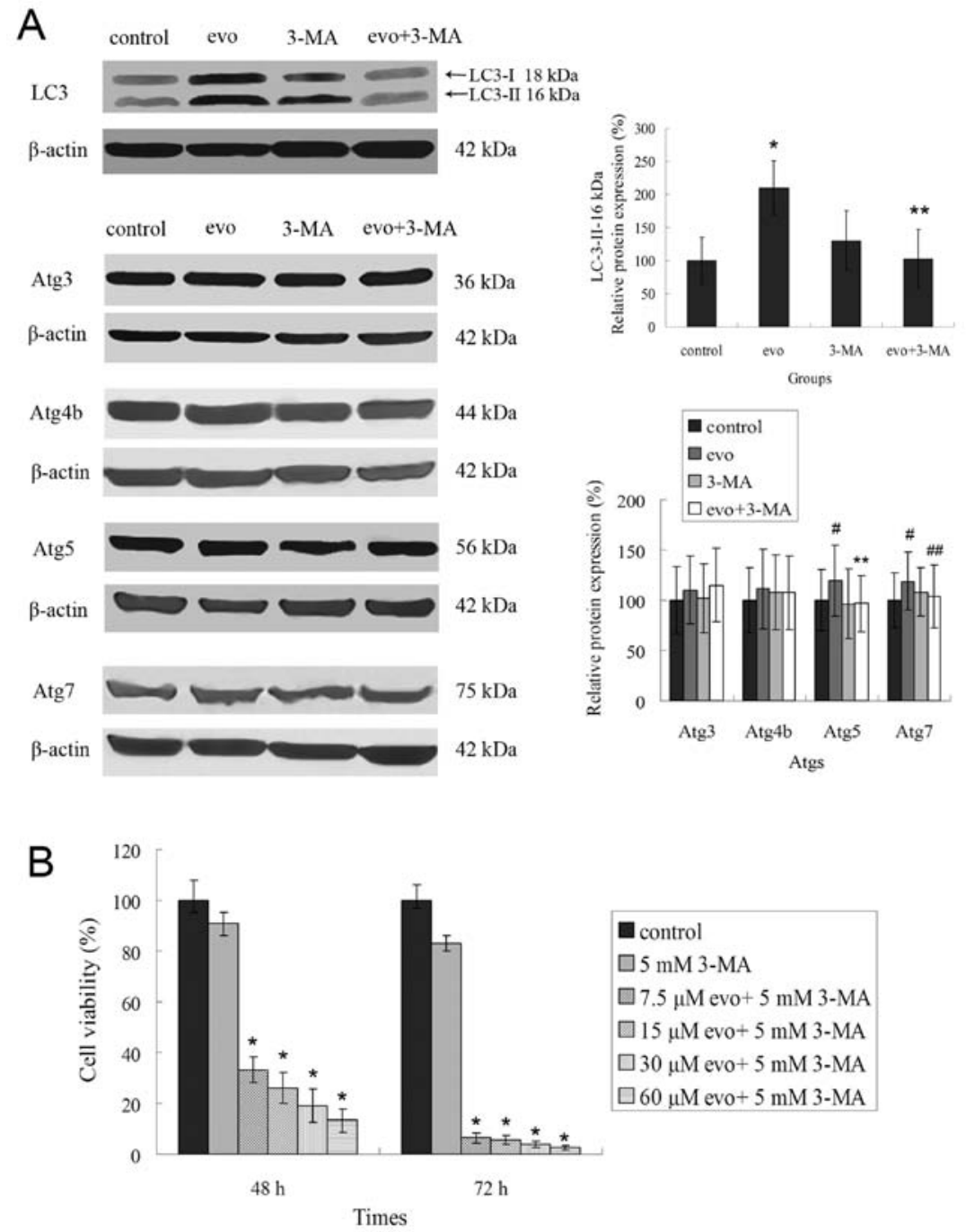

Figure 6. Inhibition of autophagy enhances evodiamine-induced cell death. (A) LLC cells were treated with the indicated agents for $1.5 \mathrm{~h}$, the levels of LC3, Atg3, Atg $4 \mathrm{~b}, \mathrm{Atg} 5$ and Atg7 were detected by western blot analysis. $\beta$-actin was used as an equal loading control. Data are expressed as means $\pm \mathrm{SD},(\mathrm{n}=3)$. ${ }^{*} \mathrm{P}<0.01$ and ${ }^{\#} \mathrm{P}<0.05$ vs. the control group, ${ }^{* *} \mathrm{P}<0.01$ and ${ }^{\# \#} \mathrm{P}<0.05$ vs. the evodiamine treatment group. (B) LLC cells were treated with the indicated agents for 48 and $72 \mathrm{~h}$. Cell viability was measured by the MTT assay. Data are presented as the percentage by control, which was defined as $100 \%(\mathrm{n}=5)$ and means \pm SD. ${ }^{*} \mathrm{P}<0.01 \mathrm{vs}$. the control group.

During our study, evodiamine also induced autophagy of LLC cells. Autophagy is a ubiquitous physiological process and is induced in nutrient or growth factor deficient conditions. Autophagosomes degrade cytoplasm and some organelles to provide nutrients and energy for cell survival (26). Autophagosome-mediated degradation is dependent upon two conjugation systems reminiscent of the ubiquitination proteosomal pathway. Atg12 is conjugated to Atg5 by the combined action of Atg7 and Atg10 (E1 and E2-like enzymes, respectively). The final complex is formed by Atg5-Atg12 non-covalently associated with the coiled-coil protein Atg 16 . This system initiates the formation of the sequestering membrane $(27,28)$. The second ubiquitin-related system leads to the conjugation of LC3 (the homologue of Atg8 in yeast) to the lipid PE by Atg7, Atg4b and Atg3. The lapidated form of LC3 is referred to as LC3-II and localizes to autophagosomal membranes. LC3-II has been shown to be an autophagosomal marker in mammals $(29,30)$. We determined that evodiamine induces autophagy in vitro and in vivo by detecting the conversion of LC3-I to LC3-II as well as the protein expressions of Atg4b, Atg5 and Atg7.
Autophagosome formation is also regulated by other mechanisms. These include the association of class III PI3K with Beclin 1 and recruitment of the Atg12-Atg 5 complex (31). Atg12-Atg 5 conjugation is required for the elongation of the isolation membrane and for localization of conjugated LC3 (32). The inhibitor, 3-MA, inhibits autophagy by blocking the activity of class III PI3K without affecting protein synthesis or ATP levels $(33,34)$. We showed that 3-MA inhibits autophagy of evodiamine-treated LLC cells, and decreases the conversion of LC3-I to LC3-II and expression of Atg7 and Atg5. Consistent with this result, conversion of LC3-I to LC3-II was also attenuated by the combinatorial treatment, evodiamine and 3-MA, in vivo.

Since autophagy is under the control of several tumorsuppressor proteins, such as Beclin 1 (35) and PTEN (36), researchers hypothesized that the autophagy process decrease is associated with tumor progression. However, autophagy can be induced by chemotherapy including cisplatin (18), anthocyanin (19), 5-fluorouracil $(20,21)$, dexamethasone (37), sulforaphane (38), curcumin (39), oridonin (40), bafilomycin $A_{1}$ 
A

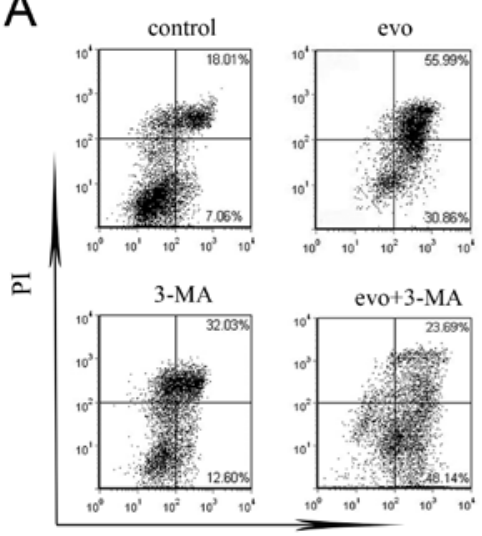

Annexin V-FITC

B

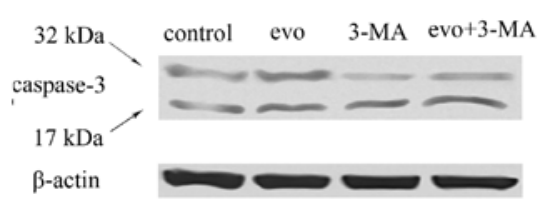

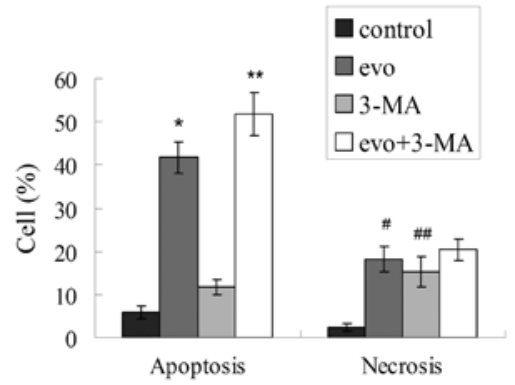

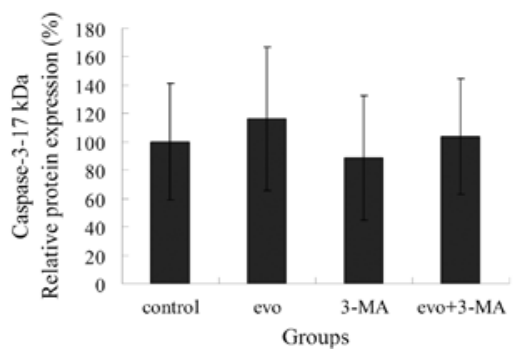

Figure 7. 3-MA augments evodiamine-induced apoptosis in LLC cells. LLC cells were treated with the indicated agents for $48 \mathrm{~h}$. (A) Apoptosis was measured by flow cytometry. Data are expressed as means $\pm \mathrm{SD},(\mathrm{n}=3)$. ${ }^{*} \mathrm{P}<0.01$ and ${ }^{\#} \mathrm{P}<0.05$ vs. the control group, ${ }^{* *} \mathrm{P}<0.01$ and ${ }^{\# \#} \mathrm{P}<0.05$ vs. the evodiamine group. $(\mathrm{B})$ Total protein was isolated and the protein expression of caspase- 3 was detected by western blot analysis. $\beta$-actin was used as an equal loading control.

A

controi

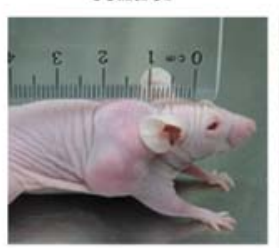

3-MA

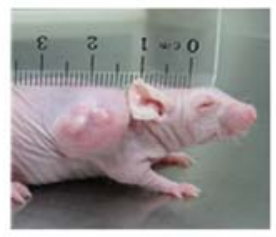

evo+3-MA

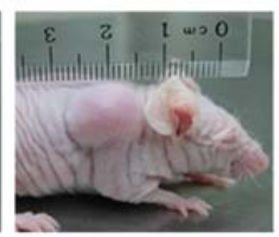

B

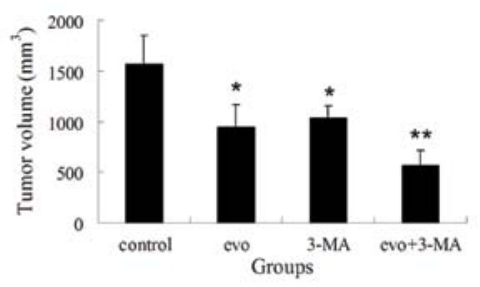

C

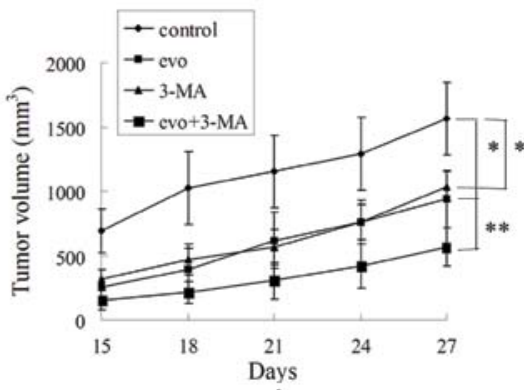

D

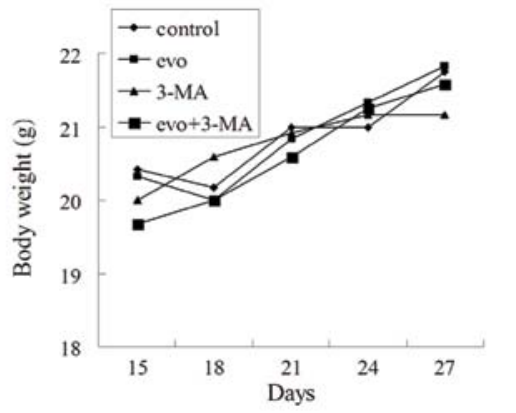

Figure 8. Anticancer effect of evodiamine and 3-MA combination against LLC xenografts. Mice were subcutaneously administered $2 \times 10^{6} \mathrm{LLC}$ cells in $0.1 \mathrm{ml}$ of PBS. Two weeks later, mice were randomly divided into the DMSO, 3-MA, evodiamine and evodiamine + 3-MA groups. Agents were injected intratumorally every 3 days for a total of 5 times (Days 15, 18,21,24 and 27) and the tumor volume and body weight of these mice were measured. The tumor volume was determined by the following formula: $0.5 \mathrm{xLxW}^{2}$ (L, length; $\mathrm{W}$, width). (A) Representative images of tumor-bearing mice and tumors. (B) The tumor volume of the final measurement. (C and D) The tumor growth curve and body weight curve. Data are expressed as means $\pm \mathrm{SD},(\mathrm{n}=8)$. ${ }^{*} \mathrm{P}<0.01$ vs. the control group, ${ }^{* * *} \mathrm{P}<0.01$ vs. the evodiamine treatment group. 
A
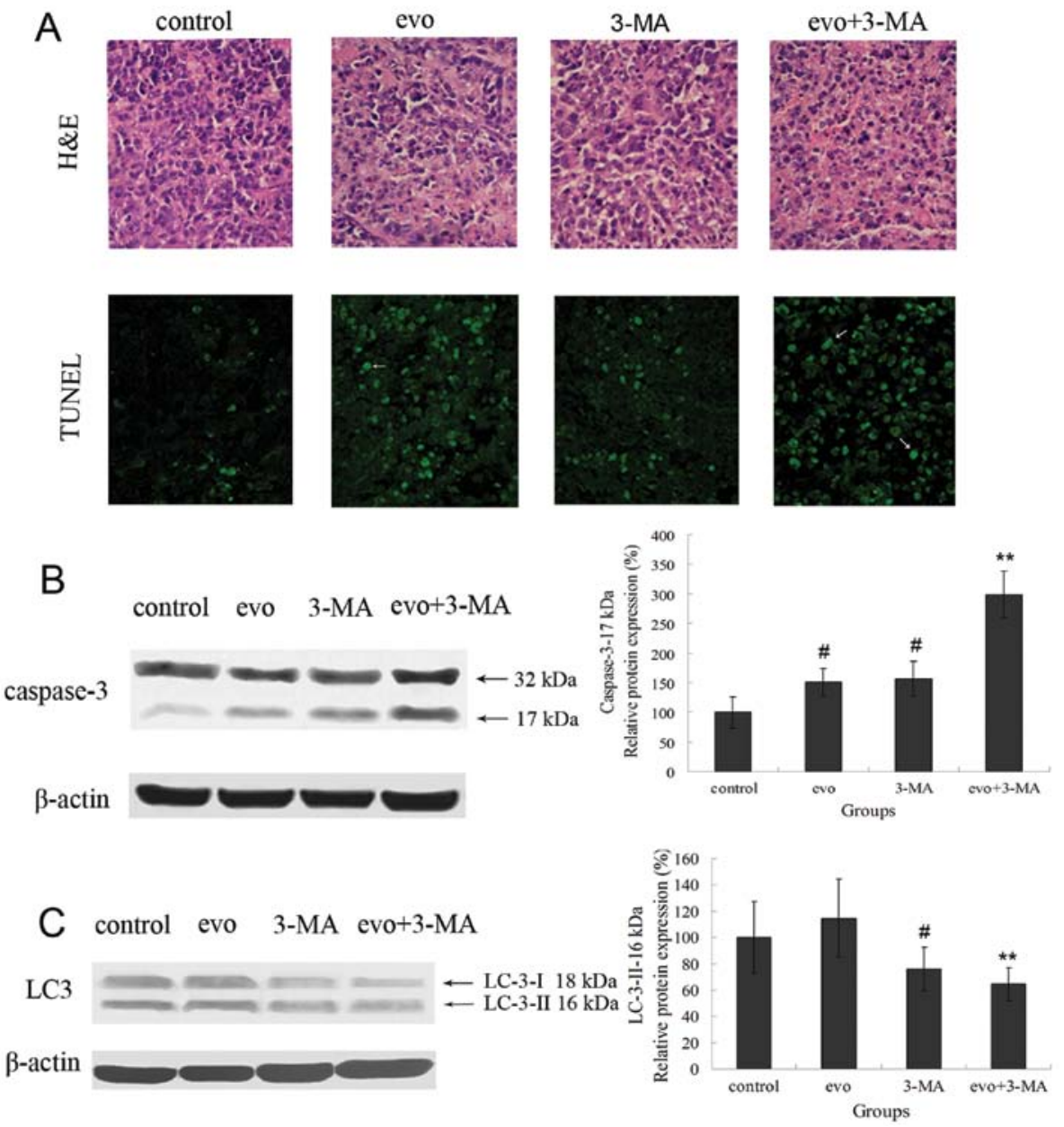

Figure 9. Evodiamine and 3-MA combination treatment enhances caspase-dependent apoptosis in vivo. (A) The tumor tissue sections were deparaffinized in xylene, and dehydrated in gradient ethanol. Then, these sections were stained by H\&E or TUNEL staining and representative images were captured (x400). The white arrow indicates TUNEL-positive cells. (B and C) Tumors were lysed and the protein was extracted, and the expressions of caspase-3 and LC 3 were then measured by western blot analysis. The amount of $\beta$-actin was used as an equal loading control. Data are expressed as means $\pm \mathrm{SD}$, ( $\mathrm{n}=8$ ). ${ }^{\#} \mathrm{P}<0.05 \mathrm{vs}$. the control group, ${ }^{* *} \mathrm{P}<0.01$ vs. the evodiamine treatment group.

(41), and resveratrol (42). Although autophagy can serve as a protective response and inhibit apoptosis, it can also enhance cell death. In this study, evodiamine combined with 3-MA was much more potent for suppressing cell proliferation than evodiamine alone in vitro and in vivo. This indicated that evodiamine-induced autophagy was protective against the cytotoxicity of evodiamine on LLC cells. Autophagy inhibition improved the sensitivity of LLC cells to evodiamine, and cell death was increased. Furthermore, the apoptotic and autophagic pathways are not mutually exclusive as they have been shown to act in synergy (43). However, the exact role and the relationship between autophagy and apoptosis in cancer remain unknown. Our findings revealed that using both evodiamine and 3-MA enhances cell apoptosis in vitro and in vivo. Although in vitro treatment had no, in vivo treatment increased caspase-3 activity. We presumed that the apoptosis pathway induced by the combination treatment in vitro is different from what occurs in vivo and may be tumor microenvironment specific.

3-MA also blocks class I PI3K activity and suppresses the invasion of HT1080 cells. This may be independent of autophagic inhibition by decreasing type I and II PI3Ks and possibly other molecules (44). The PI3K/Akt signaling plays a central role in modulating cell survival and apoptosis (45).
It is possible that 3-MA also affects this signaling pathway in vitro and in vivo. In some previous studies, 3-MA was found to be cytotoxic against cancer cells $(42,46,47)$. However, its cytotoxicity in LLC cells was modest in our experiments.

Collectively, our study demonstrated that evodiamine inhibits the growth of LLC cells by increasing apoptosis in a caspase-independent manner in vitro and in a caspase-dependent pathway in vivo. Meanwhile, evodiamine enhanced the conversion of LC3-I to LC3-II, and upregulated the expression of Atg4b, Atg5 and Atg7. This induced autophagy was cytoprotective, as inhibition of evodiamine-induced autophagy by 3-MA could potentiate the cytotoxicity of evodiamine in vitro and in vivo. Although evodiamine possesses anticancer effects on multiple cancer cells, its anticancer activity has yet to be fully elucidated. Our observations provide additional insights into the therapeutic effects of evodiamine in cancer.

\section{Acknowledgements}

This study was supported by Grants from the National Natural Science Foundation of China (No. 30772253) and the Project of State Key Laboratory of Trauma, Burns, and Combined Injury (No. SKLZZ201005). 


\section{References}

1. Jemal A, Siegel R, Ward E, et al: Cancer statistics, 2008. CA Cancer J Clin 58: 71-96, 2008.

2. Petty RD, Nicolson MC,Kerr KM, et al: Gene expression profiling in non-small cell lung cancer: from molecular mechanisms to clinical application. Clin Cancer Res 10: 3237-3248, 2004.

3. Erridge SC, Moller H, Price A, et al: International comparisons of survival from lung cancer: pitfalls and warnings. Nat Clin Pract Oncol 4: 570-577, 2007.

4. Wang TY, Wu JB, Hwang TL, et al: A new quinolone and other constituents from the fruits of Tetradium ruticarpum: effects on neutrophil pro-inflammatory responses. Chem Biodivers 7: $1828-1834,2010$

5. Liu YN, Pan SL, Liao CH, et al: Evodiamine represses hypoxiainduced inflammatory proteins expression and hypoxia-inducible factor 1alpha accumulation in RAW264.7. Shock 32: 263-269, 2009.

6. Wang T, Wang Y and Yamashita H: Evodiamine inhibits adipogenesis via the EGFR-PKCalpha-ERK signaling pathway. FEBS Lett 583: 3655-3659, 2009.

7. Wu CL, Hung CR, Chang FY, et al: Effects of evodiamine on gastrointestinal motility in male rats. Eur J Pharmacol 457: 169-176, 2002.

8. Zhang C, Fan X, Xu X, et al: Evodiamine induces caspasedependent apoptosis and $\mathrm{S}$ phase arrest in human colon lovo cells. Anticancer Drugs 21: 766-776, 2010.

9. Yang J, Wu LJ, Tashino S, et al: Protein tyrosine kinase pathwayderived ROS/NO productions contribute to G2/M cell cycle arrest in evodiamine-treated human cervix carcinoma HeLa cells. Free Radic Res 44: 792-802, 2010

10. Wang C, Li S and Wang MW: Evodiamine-induced human melanoma A375-S2 cell death was mediated by PI3K/Akt/caspase and Fas-L/NF-kappaB signaling pathways and augmented by ubiquitin-proteasome inhibition. Toxicol In Vitro 24: 898-904, 2011.

11. Chen MC, Yu CH, Wang SW, et al: Anti-proliferative effects of evodiamine on human thyroid cancer cell line ARO. J Cell Biochem 110: 1495-1503, 2011.

12. Wang XN, Han X, Xu LN, et al: Enhancement of apoptosis of human hepatocellular carcinoma SMMC-7721 cells through synergy of berberine and evodiamine. Phytomedicine 15: 1062-1068, 2008.

13. Kan SF, Yu CH, Pu HF, et al: Anti-proliferative effects of evodiamine on human prostate cancer cell lines DU145 and PC3. J Cell Biochem 101: 44-56, 2007.

14. Ogasawara M, Matsunaga T, Takahashi S, et al: Anti-invasive and metastatic activities of evodiamine. Biol Pharm Bull 25: 1491-1493, 2002

15. Meijer AJ and Codogno P: Regulation and role of autophagy in mammalian cells. Int J Biochem Cell Biol 36: 2445-2462, 2004.

16. Yang YP, Liang ZQ, Gao B, et al: Dynamic effects of autophagy on arsenic trioxide-induced death of human leukemia cell line HL60 cells. Acta Pharmacol Sin 29: 123-134, 2008.

17. Ogier-Denis E and Codogno P: Autophagy: a barrier or an adaptive response to cancer. Biochim Biophys Acta 1603: 113-128, 2003.

18. Liu D, Yang Y and Liu Q: Inhibition of autophagy by 3-MA potentiates cisplatin-induced apoptosis in esophageal squamous cell carcinoma cells. Med Oncol 28: 105-111, 2011.

19. Longo L, Platini F, Scardino A, et al: Autophagy inhibition enhances anthocyanin-induced apoptosis in hepatocellular carcinoma. Mol Cancer Ther 7: 2476-2485, 2008.

20. Li J, Hou N, Faried A, et al: Inhibition of autophagy by 3-MA enhances the effect of 5-FU-induced apoptosis in colon cancer cells. Ann Surg Oncol 16: 761-771, 2009.

21. Li J, Hou N, Faried A, et al: Inhibition of autophagy augments 5 -fluorouracil chemotherapy in human colon cancer in vitro and in vivo model. Eur J Cancer 46: 1900-1909, 2010.

22. Yang J, Wu LJ, Tashino S, et al: Reactive oxygen species and nitric oxide regulate mitochondria-dependent apoptosis and autophagy in evodiamine-treated human cervix carcinoma HeLa cells. Free Radic Res 42: 492-504, 2008.

23. Lowe SW and Lin AWA: Apoptosis in cancer. Carcinogenesis 21: 485-495, 2000

24. Elmore S: Apoptosis: a review of programmed cell death. Toxicol Pathol 35: 495-516, 2007
25. Van Gurp M, Festjens N, Van Loo G, et al: Mitochondrial intermembrane proteins in cell death. Biochem Biophys Res Commun 304: 487-497, 2003.

26. Levine B and Klionsky DJ: Development by self-digestion: molecular mechanisms and biological functions of autophagy. Dev Cell 6: 463-477, 2004.

27. Kuma A, Mizushima N, Ishihara N, et al: Formation of the approximately 350-kDa Apg12-Apg5.Apg16 multimeric complex, mediated by Apg16 oligomerization, is essential for autophagy in yeast. J Biol Chem 277: 18619-18625, 2002.

28. Fujita N, Itoh T, Omori H, et al: The Atg16L complex specifies the site of LC3 lipidation for membrane biogenesis in autophagy. Mol Biol Cell 19: 2092-2100, 2008.

29. Kabeya Y, Mizushima N, Ueno T, et al: LC3, a mammalian homologue of yeast Apg8p, is localized in autophagosome membranes after processing. EMBO J 19: 5720-5728, 2000.

30. Hemelaar J, Lelyveld VS, Kessler BM and Ploegh HL: A single protease, $\mathrm{Apg} 4 \mathrm{~B}$, is specific for the autophagy-related ubiquitinlike proteins GATE-16, MAP1-LC3, GABARAP, and Apg8L. J Biol Chem 278: 51841-51850, 2003.

31. Suzuki K, Kirisako T, Kamada Y, et al: The pre-autophagosomal structure organized by concerted functions of APG genes is essential for autophagosome formation. EMBO J 20: 5971-5981, 2001.

32. Mizushima N, Yamamoto A, Hatano M, et al: Dissection of autophagosome formation using Apg5-deficient mouse embryonic stem cells. J Cell Biol 152: 657-668, 2001.

33. Seglen PO and Gordon PB: 3-Methyladenine: specific inhibitor of autophagic/lysosomal protein degradation in isolated rat hepatocytes. Proc Natl Acad Sci USA 79: 1889-1892, 1982

34. Petiot A, Ogier-Denis E and Blommaart EF: Distinct classes of phosphatidylinositol 3'-kinases are involved in signaling pathways that control macroautophagy in HT-29 cells. J Biol Chem 275: 992-998, 2000.

35. Maiuri MC, Criollo A and Kroemer G: Crosstalk between apoptosis and autophagy within the Beclin 1 interactome. EMBO J 29: 515-516, 2010.

36. Gozuacik D and Kimchi A: Autophagy as a cell death and tumor suppressor mechanism. Oncogene 23: 2891-2906, 2004.

37. Laane E, Tamm KP, Buentke E, et al: Cell death induced by dexamethasone in lymphoid leukemia is mediated through initiation of autophagy. Cell Death Differ 16: 1018-1029, 2009.

38. Herman-Antosiewicz A, Johnson DE and Singh SV: Sulforaphane causes autophagy to inhibit release of cytochrome $\mathrm{C}$ and apoptosis in human prostate cancer cells. Cancer Res 66: 5828-5835, 2006

39. Aoki H, Takada Y, Kondo S, et al: Evidence that curcumin suppresses the growth of malignant gliomas in vitro and in vivo through induction of autophagy: role of Akt and extracellular signal-regulated kinase signaling pathways. Mol Pharmacol 72: 29-39, 2007.

40. Cui Q, Tashiro S, Onodera S, et al: Autophagy preceded apoptosis in oridonin-treated human breast cancer MCF-7 cells. Biol Pharm Bull 30: 859-864, 2007.

41. Wu YC, Wu WK, Li Y, et al: Inhibition of macroautophagy by bafilomycin A1 lowers proliferation and induces apoptosis in colon cancer cells. Biochem Biophys Res Commun 382: 451-456, 2009.

42. Hsu KF, Wu CL, Huang SC, et al: Cathepsin L mediates resveratrol-induced autophagy and apoptotic cell death in cervical cancer cells. Autophagy 5: 451-460, 2009.

43. Eisenberg-Lerner A, Bialik S, Simon HU, et al: Life and death partners: apoptosis, autophagy and the cross-talk between them. Cell Death Differ 16: 966-975, 2009.

44. Ito S, Koshikawa N, Mochizuki S, et al: 3-Methyladenine suppresses cell migration and invasion of HT1080 fibrosarcoma cells through inhibiting phosphoinositide 3-kinases independently of autophagy inhibition. Int J Oncol 31: 261-268, 2007.

45. Goswami A, Ranganathan P and Rangnekar VM: The phosphoinositide 3-kinase/Akt1/Par-4 axis: a cancer-selective therapeutic target. Cancer Res 66: 2889-2892, 2006.

46. Opipari AW Jr, Tan L, Boitano AE, et al: Resveratrol-induced autophagocy tosis in ovarian cancer cells. Cancer Res 64: 696-703, 2004.

47. Trincheri NF, Follo C, Nicotra G, et al: Resveratrol-induced apoptosis depends on the lipid kinase activity of $\mathrm{Vps} 34$ and on the formation of autophagolysosomes. Carcinogenesis 29: 381-389, 2008. 